\title{
Antidiabetic Drugs in Combination with Hydroxychloroquine Improve Glycemic Control in Alloxan Induced Diabetic Rats
}

\author{
Shaheda Zannah', Md. Saiful Islam¹, A. F. M. Towheedur Rahman1, Md. Asaduzzaman1, \\ Abdul Alim Al Bari'1, Yusuf Ali', Gazi Jahirul Islam², A. H. M. Khurshid Alam¹, Hazrat Ali', \\ Mamunur Rashid1* \\ ${ }^{1}$ Department of Pharmacy, University of Rajshahi, Rajshahi, Bangladesh \\ ${ }^{2}$ Department of Chemistry, Khulna University of Engineering and Technology, Khulna, Bangladesh \\ Email: ${ }^{*}$ mamun69jp@yahoo.com
}

Received 2 May 2014; revised 3 June 2014; accepted 17 June 2014

Copyright (C) 2014 by authors and Scientific Research Publishing Inc. This work is licensed under the Creative Commons Attribution International License (CC BY). http://creativecommons.org/licenses/by/4.0/ (c) (i) Open Access

\section{Abstract}

Worldwide prevalence of diabetes mellitus has become an issue of great concern in current decades. This life threatening disease is associated with worsening of glycemic control and progressive metabolic dysfunctions. Objective: Current study aimed to investigate the effect of hydroxychloroquine (HCQ) as an adjunct to glibenclamide or metformin on glycemic control in alloxan induced diabetic rats. Methods: HCQ was combined separately with two conventional anti-diabetic drugs; glibenclamide and metformin. At first, alloxan $(120 \mathrm{mg} / \mathrm{kg})$ induced diabetic rats were treated with single dose of metformin $(850 \mathrm{mg} / 70 \mathrm{~kg} \mathrm{BW})$, glibenclamide $(10 \mathrm{mg} / 70 \mathrm{~kg} \mathrm{BW})$ and HCQ $(300 \mathrm{mg} / 70 \mathrm{~kg} \mathrm{BW})$ intraperitoneally once daily for two weeks. Then non fixed dose combinations of glibenclamide $(5 \mathrm{mg} / 70 \mathrm{~kg} \mathrm{BW})$ with HCQ $(150 \mathrm{mg} / 70 \mathrm{~kg} \mathrm{BW})$ and metformin $(425$ $\mathrm{mg} / 70 \mathrm{~kg} \mathrm{BW})$ with HCQ $(150 \mathrm{mg} / 70 \mathrm{~kg} \mathrm{BW})$ were injected along with those of the three drugs alone once daily for four weeks. Results: In alloxan induced diabetic rats, glibenclamide, metformin and their combination therapies reduced blood glucose level significantly but combination therapies are the most effective. Glibenclamide or metformin in combination with HCQ also significantly $(P<0.05)$ reduced the elevated levels of total cholesterol, triglycerides, and low density lipoprotein cholesterol (LDL-C) level and increased high density lipoprotein cholesterol (HDL-C) level. Moreover, HCQ potentiates the liver glycogen synthesis of metformin or glibenclamide. Conclusion: Outcomes of this investigation indicate that combination of glibenclamide or metformin with HCQ improves glycemic control and provides additional metabolic benefits, not achieved

\footnotetext{
${ }^{*}$ Corresponding author.
}

How to cite this paper: Zannah, S., et al. (2014) Antidiabetic Drugs in Combination with Hydroxychloroquine Improve Glycemic Control in Alloxan Induced Diabetic Rats. Pharmacology \& Pharmacy, 5, 725-735. 
with either glibenclamide or metformin alone.

\author{
Keywords
}

\author{
Diabetes Mellitus, Glycemic Control, HCQ, Glibenclamide, Metformin
}

\title{
1. Introduction
}

An intensive treatment of hyperglycemia is known to lower the risks of microvascular complications such as retinopathy, nephropathy, and neuropathy as well as the incidence of cardiovascular events in both Type 1 and Type 2 diabetic patients [1] [2]. Unfortunately, many diabetic patients hardly achieve and/or maintain the recommended $7 \%$ glycated hemoglobin $\left(\mathrm{HbA}_{1 \mathrm{c}}\right)$ goal for blood glucose control and are therefore exposed to increasing risks of diabetic complications as glycemic control deteriorates over time [3]. Studies have shown that diabetes mellitus is a progressive disorder which cannot be effectively managed with single drug therapy [4] [5]. Regardless of drug management, the pancreatic $\beta$-cells in type 2 diabetic patients continue to deteriorate, leading to the worsening of glycemic control and consequent requirement for multiple therapies or exogenous insulin [6].

Increased generation of reactive oxygen species (ROS) resulting from metabolism of excessive glucose and/or free fatty acid has been identified as a contributor to the deterioration of pancreatic $\beta$-cell function [7] [8]. Due to the relatively low expression of antioxidant enzymes such as superoxide dismutase, catalase and glutathione peroxidase in the pancreas [9], the pancreatic $\beta$-cells may be highly vulnerable to damage when exposed to oxidative stress. Thus, oxidative stress may play a major role in $\beta$-cell deterioration frequently observed in Type 2 diabetes [10].

Often patients on combination therapy with sulphonylureas and metformin are not controlled and hence the option left is either addition of third drug or put patient on insulin therapy. Insulin therapy is not only costly but is not preferred due to poor patients' compliance in parenteral application. New generation thiazolidinediones class of antidiabetic drugs, though useful in glycemic control, are associated with several adverse effects such as excessive risk of congestive heart failure, acute myocardial infarction, increased rate of bone loss and liver toxicity.

In the light of failure of mono-therapy of anti-diabetic drugs in glycemic control and increased adverse effects when administered at high doses of anti-diabetic drugs for getting better glycemic control, newer medications for diabetes are needed, which will have good anti-hyperglycemic effect, as well as good tolerability profile.

HCQ is an anti-malarial drug that may have anti-hyperglycemic properties in patients with Type 2 diabetes mellitus and the beneficial effects on glucose metabolism and insulin sensitivity was reported among patients with systemic Lupus Erythematousus [11] [12]. HCQ improves glycemic control in sulphonylureas refractory patients with poorly controlled Type 2 diabetes [11]. The addition of HCQ to insulin therapy caused a significant decrease in the insulin requirements [11]. This effect appeared at 2 weeks and persisted for 6 months. In a prospective, multicentre, observational study, Wasko et al. [13] showed that 4905 rheumatoid arthritis (RA) patients using HCQ were associated with a reduced risk of diabetes.

In the present study, we have investigated how the addition of HCQ as an adjunct to glibenclamide or metformin affects glycemic control in alloxan induced diabetic rats.

In the light of the above data, the objectives of the present study were to evaluate the effect of HCQ on blood glucose and lipid profile in alloxan induced diabetic rats and to study its effect on the above parameters in combination with conventional oral antidiabetic agents.

\section{Materials and Methods}

\subsection{Selection of Animal}

Long Evans male rats weighing about 150 - 180 gm, age 2 months were acclimatized to the new environmental condition for a period of one week. During the experimental period the rats were kept in a well ventilated animal house at room temperature of $25^{\circ} \mathrm{C}$ and were supplied with standard pellets from ICDDR, B and fresh drinking water. All the rats were kept in cages and maintained with natural 12-hour light and dark cycle. All protocols for the animal experiments were assessed and endorsed by the Animal Care and Use Committee of Institute of Biological Sciences, University of Rajshahi, Bangladesh. 


\subsection{Experimental Induction of Diabetes}

Long Evans male rats except normal control group were allowed to fast for 12 hours. Hyperglycemia was induced in each fasted rat by administering alloxan monohydrate $(120 \mathrm{mg} / \mathrm{kg}$ body weight) in normal saline intraperitoneally. After 24 hours, blood glucose content was measured by using Clever Check glucose test meter (Bioland, Germany) using blood sample collected from the tail vein of the rats. When the condition of diabetes was established animals with blood glucose levels above $11.1 \mathrm{mmol} / \mathrm{L}$ was selected for the study [14].

\subsection{Preparation of HCQ, Glibenclamide and Metformin}

Two hypoglycemic drugs (metformin and glibenclamide) were used in the present study as reference standard drug. Each drug was administered intra-peritoneally to hyperglycemic rats $(n=6)$. Metformin was administered at $850 \mathrm{mg} / 70 \mathrm{~kg}$ body weight/day and glibenclamide at $10 \mathrm{mg} / 70 \mathrm{~kg}$ body weight/day. The trial drug hydroxychloroquine was administered at $300 \mathrm{mg} / 70 \mathrm{~kg}$ body weight/day. Glibenclamide and metformin were dissolved in dimethyl sulfoxide and distilled water respectively before they were administered. HCQ was dissolved in distilled water. In case of combination therapy, metformin with HCQ was prepared in solution form according to the dose of (425 mg/70kg BW $+150 \mathrm{mg} / 70 \mathrm{~kg} \mathrm{BW})$ and glibenclamide with HCQ was prepared according to the dose of (5 mg/70kg BW + $150 \mathrm{mg} / 70 \mathrm{~kg} \mathrm{BW})$ respectively.

\subsection{Treatment}

The animals were randomly divided into seven groups. Each group comprised six rats. The treatment of animals began on the 3rd day after alloxan injection and this was considered as 1st day of treatment. At the end of the treatment period, the animals were fasted for at least 16 hours and sacrificed. Blood samples were collected in centrifuge tubes without anticoagulants and allowed to clot. The clotted blood was then centrifuged at $4000 \mathrm{rpm}$ for $30 \mathrm{~min}$. Serum was separated and then quickly stored at refrigerator for biochemical analyses.

\subsection{Two Weeks Treatment Protocol}

Glibenclamide, metformin and HCQ were administered daily for two weeks in alloxan induced diabetic rats. After two weeks treatment blood glucose was determined two hours after last dose using glucometer. In addition serum lipid profiles were also assessed. All assays were carried out using diagnostic kits.

\subsection{Four Weeks Treatment Protocol}

Glibenclamide, metformin, HCQ and combination (glibenclamide with HCQ) and (metformin with HCQ) were administered daily for four weeks in the alloxan induced diabetic rats. After four weeks treatment, blood glucose was determined two hours after last dose using glucometer. In addition, serum lipid profiles were also assessed. All assays were carried out using diagnostic kits.

\subsection{Biochemical Analyses}

Serum glucose was determined by the glucose oxidase method using Clever Check glucose test meter (Bioland, Germany). Serum high density lipoprotein cholesterol (HDL-C) was estimated by the UV spectrophotometric method, using HDL CHOLESTEROL liquicolor test kit. Serum low density lipoprotein cholesterol (LDL-C) was estimated by the method of UV spectrophotometric method, using CHOLESTEROL LDL REAGENT PRECIPITATE test kit. Serum total cholesterol (TC) and triglycerides (TG) were estimated by the methods of UV spectrophotometric, using CHOLESTEROL liquicolor and TRIGLYCERIDES liquicolor test kits.

Glycogen content in liver was measured according to spectrophotometric determination of glycogen with otoluidine reagent. It utilizes the o-toluidine glucose coupling reactions for the estimation of glycogen after trichloroacetic acid extraction, precipitation by alcohol and hydrolysis.

\subsection{Statistical Analysis}

The results were expressed as mean \pm SEM. We used a one-way analysis of variance (ANOVA), followed by Dunnett's post-hoc test or students paired or unpaired $t$-test where appropriate. The statistical method applied in each analysis was described in each figure. Results were considered to be significant when $P$ values were less 
than $0.05(P<0.05)$.

\section{Results}

The effects of drug alone (metformin/glibenclamide/HCQ) and combination (metformin with HCQ and glibenclamide with HCQ) on the parameters of blood glucose level, lipid profile (TC, TG, LDL-C, HDL-C) and liver glycogen were performed for both short and long-term alloxan induced diabetes rats.

\subsection{Effects of Metformin, Glibenclamide and HCQ on Blood Glucose Level in Short Term Alloxan Induced Diabetic Rats}

Short term intraperitoneal (IP) injection of alloxan in rats significantly increased blood glucose level (21.795 \pm $0.705 \mathrm{mmol} / \mathrm{L}$ ) when compared with normal rats ( $5.35 \pm 0.2 \mathrm{mmol} / \mathrm{L}$ ) (Figure 1$)$. There was a significant elevation in serum glucose in the diabetic animals. The effect of administration of metformin and glibenclamide tended to bring the parameter significantly towards normal values. On the other hand, HCQ did not produce any significant result after two weeks treatment.

\subsection{Effects of Metformin, Glibenclamide and HCQ on Lipid Profile in Short Term Alloxan Induced Diabetic Rats}

Short term induction of diabetes by alloxan in rats significantly altered lipid profile when compared with normal rats (Figures 2(a)-2(d)). There was significant elevation in serum TC (36.65\%), TG (41.98\%), LDL-C (33.53\%) in the diabetic animals. On the contrary, HDL-C level (42.86\%) was reduced in alloxan induced diabetic rats in comparison with their respective normal rats after two weeks. The effect of administration of metformin and glibenclamide tended to bring the TC level and HDL-C significantly towards normal values. Metformin therapy significantly reduced TG level $(P<0.05)$. While insignificant decrease was observed with glibenclamide therapy in serum triglycerides, LDL-cholesterol as depicted in Figures 2(a)-2(d). Both showed insignificant improve in case of LDL-C level. On the other hand, HCQ couldn't show any significant $(P<0.05)$ result to improve these parameters.

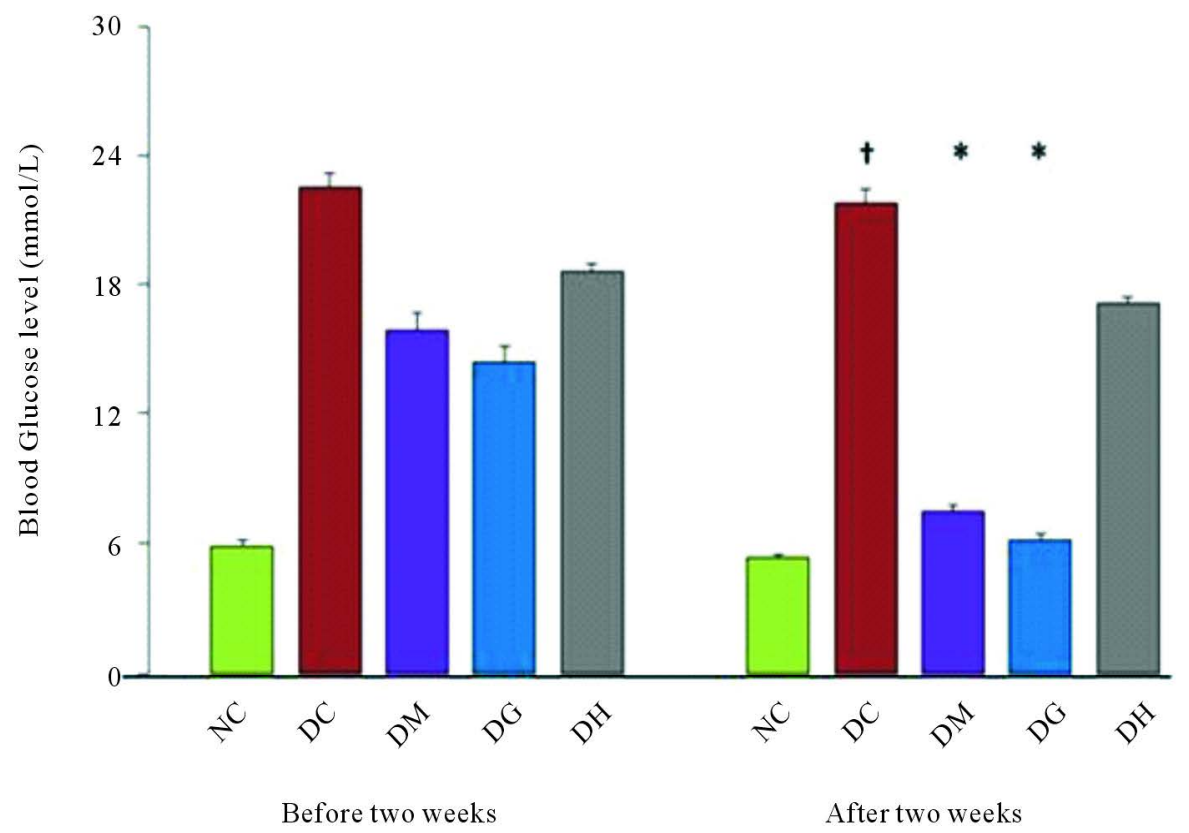

Figure 1. Effects of glibenclamide, metformin and HCQ on the fasting blood glucose level in alloxan induced diabetic rats after two weeks treatment. The values are expressed as mean \pm SEM. $n=6$ in each group. ${ }^{\dagger} P<0.05$ compared with normal control group. ${ }^{*} P<0.05$ compared with diabetic control group. NC (Normal control), DC (Diabetic control), DM (Diabetic + Metformin), DG (Diabetic + Glibenclamide), DH (Diabetic + HCQ). 


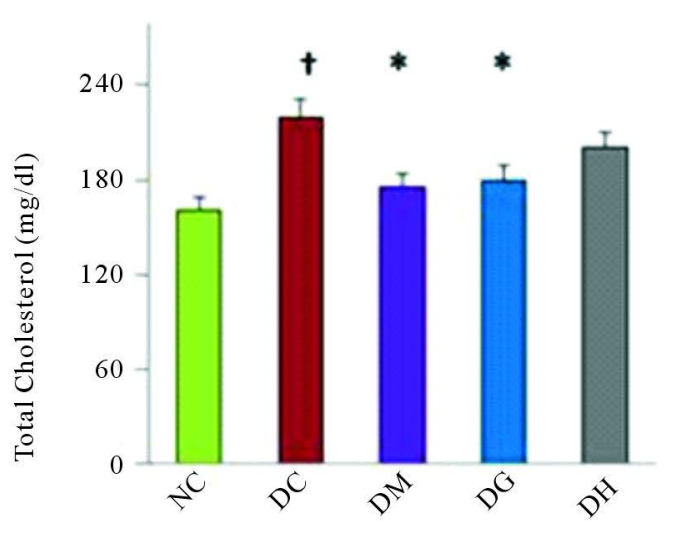

(a)

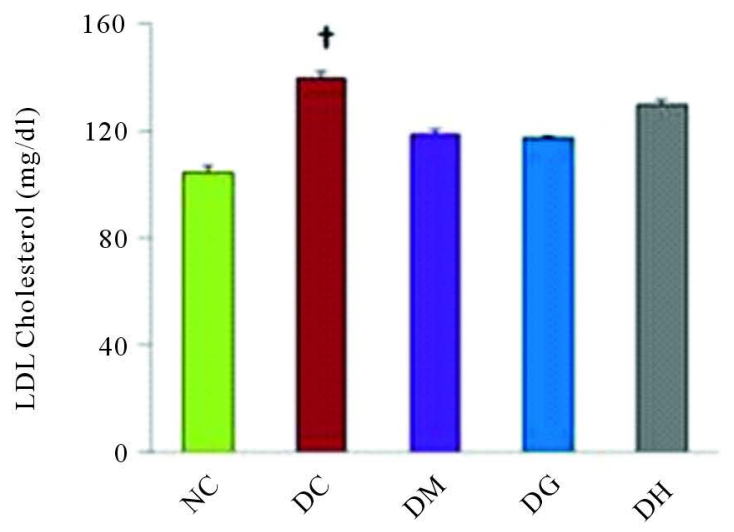

(c)

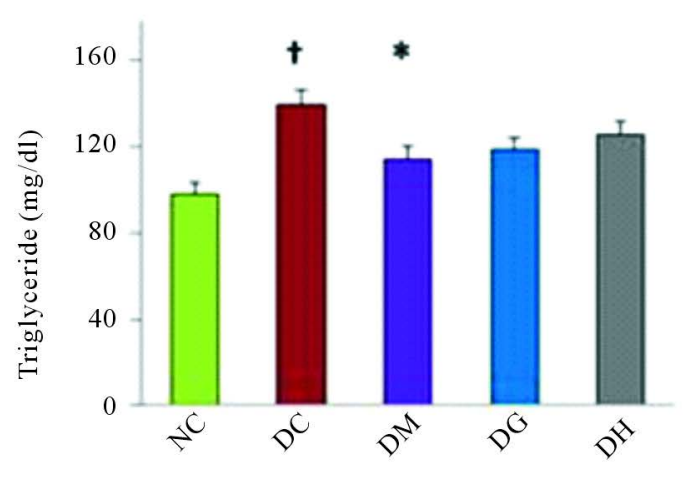

(b)

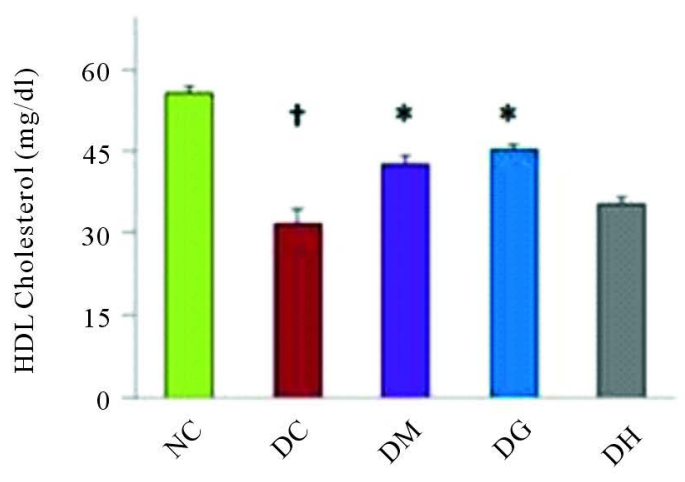

(d)

Figure 2. Effects of glibenclamide, metformin and HCQ on lipid profile in alloxan induced diabetic rats after two weeks treatment. Changes in (a) TC level, (b) TG level, (c) LDL-C level and (d) HDL-C level. All values were presented as mean \pm SEM; $n=6$ in each group. ${ }^{\dagger} P<0.05$ compared with normal control group. ${ }^{*} P<0.05$ compared with diabetic control group. NC (Normal control), DC (Diabetic control), DG (Diabetic + Glibenclamide), DM (Diabetic + Metformin), DH (Diabetic + HCQ).

\subsection{Effects of Metformin, Gibenclamide and HCQ on the Level of Glycogen Content in Short Term Alloxan Induced Diabetic Rats}

In this study, it was found that the level of glycogen in liver was reduced to $46.09 \%$ in diabetic rats as compared to the normal control group after two weeks treatment. Treatment of diabetic rats with metformin, glibenclamide and HCQ the level of glycogen content was improved to $40.58 \%, 47.8 \%$ and $15.36 \%$ respectively as shown in the Figure 3. In this case, glibenclamide had more significant activity in glycogen synthesis.

\subsection{Effects of Combination Therapy on Blood Glucose Level in Long-Term Alloxan Induced Diabetic Rats}

Long-term induction of diabetes by alloxan in rats increased blood glucose level (26.75 $\pm 0.25 \mathrm{mmol} / \mathrm{L})$ when compared with normal rats (Figure 4). To investigate the effect of mono and combination therapy on blood glucose level in long-term alloxan induced diabetic rats, we examined blood glucose level after four weeks treatment with metformin, glibenclamide, HCQ alone and combination of drugs (metformin with HCQ) and (glibenclamide with HCQ) in diabetic rats. After four weeks treatment metformin, glibenclamide and their combination of metformin with HCQ and glibenclamide with HCQ significantly $(P<0.05)$ decreased blood glucose level from $26.75 \pm 0.25$ to $7.9 \pm 0.2 \mathrm{mmol} / \mathrm{L}, 26.75 \pm 0.25$ to $7.2 \pm 0.275 \mathrm{mmol} / \mathrm{L}, 26.75 \pm 0.25$ to $6.7 \pm$ $0.125 \mathrm{mmol} / \mathrm{L}$ and $26.75 \pm 0.25$ to $6.1 \pm 0.3 \mathrm{mmol} / \mathrm{L}$ respectively. After four-week treatment metformin alone, glibenclamide alone and combination therapy (metformin with HCQ) \& (glibenclamide with HCQ) 


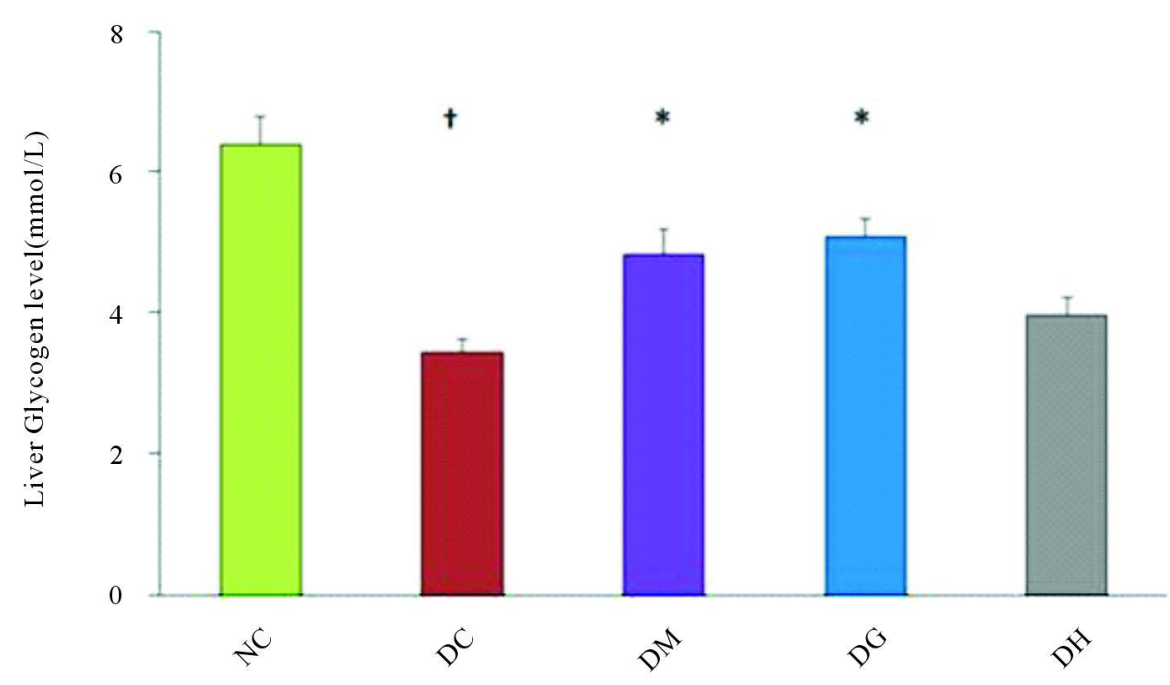

Figure 3. Effects of glibenclamide, metformin and HCQ on liver glycogen level in alloxan induced diabetic rats after two weeks treatment. All values were presented as mean \pm SEM; $n$ $=6$ in each group. ${ }^{\dagger} P<0.05$ compared with normal control group. $* P<0.05$ compared with diabetic control group. NC (Normal control), DC (Diabetic control), DG (Diabetic + Glibenclamide), DM (Diabetic + Metformin), DH (Diabetic + HCQ).

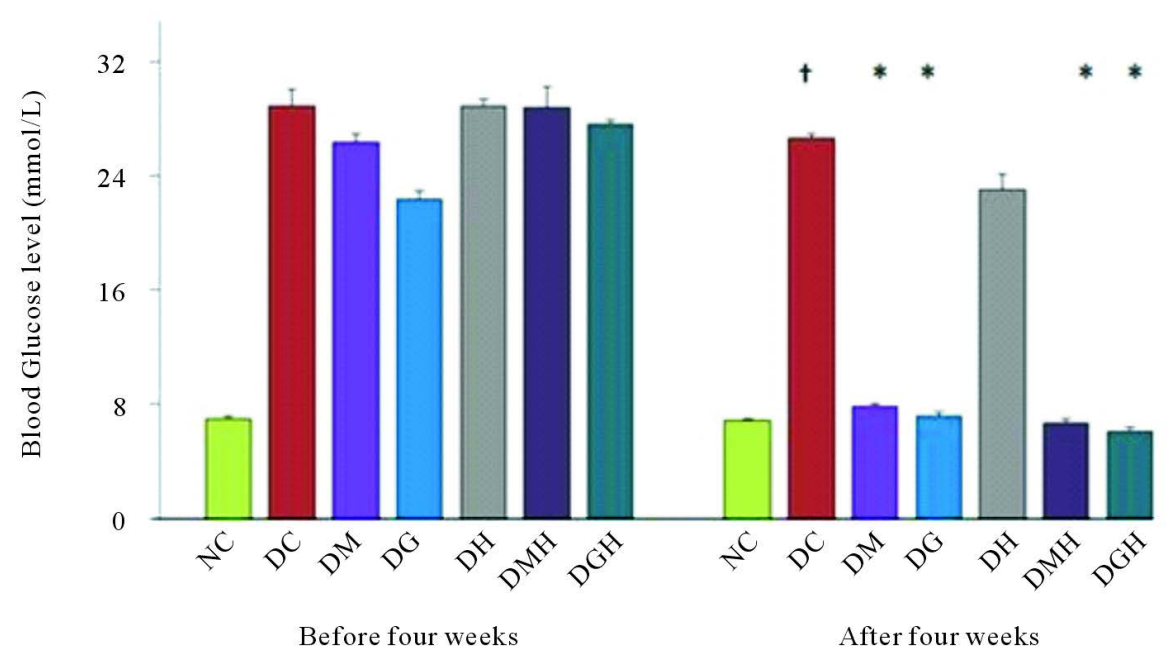

Figure 4. Effect of glibenclamide, metformin, HCQ and combination of drugs on the FBG level in alloxan induced diabetic rats after four weeks treatment. The values are expressed as mean \pm SEM. $n=6$ in each group. ${ }^{\dagger} P<0.05$ compared with normal control group. ${ }^{*} P<0.05$ compared with diabetic control group. NC (Normal control), DC (Diabetic control), DG (Diabetic + Glibenclamide), DM (Diabetic + Metformin), DH (Diabetic + HCQ), DGH (Diabetic + Glibenclamide + HCQ), DMH (Diabetic + Metformin + HCQ).

produced significant $(P<0.05)$ decrease in blood glucose level to $70.46 \%, 73.08 \%, 74.94 \%$ and $77.19 \%$ respectively in alloxan induced diabetic rats. Here the maximum reduction of blood glucose level by $77.19 \%$ was observed for (glibenclamide with HCQ). On the contrary, HCQ alone (13.64\%) did not decrease significantly blood glucose level in alloxan-induced diabetic rats.

\subsection{Effects of Combination Therapy on Lipid Profile in Long-Term Alloxan Induced Diabetic Rats}

Figures 5(a)-5(d) show the results of serum TC, serum TG, serum LDL-C and HDL-C level in different groups 


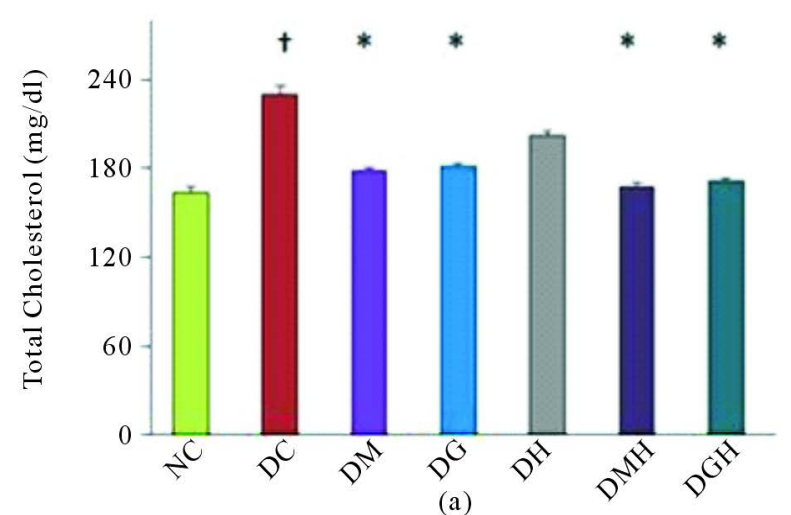

(a)

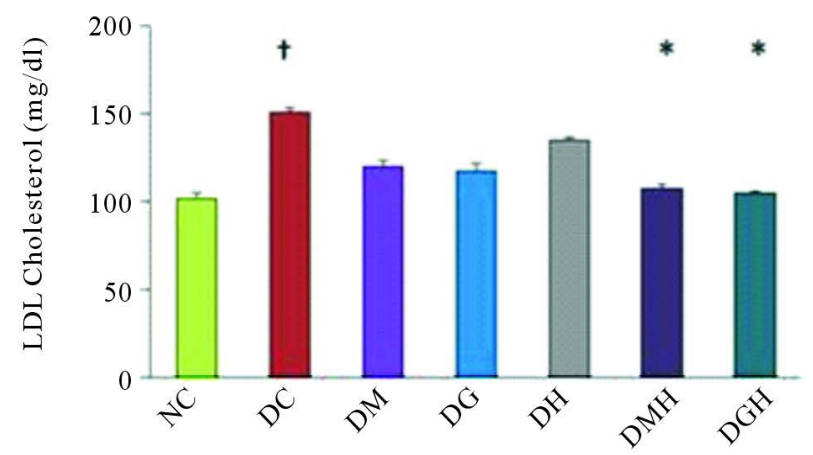

(c)

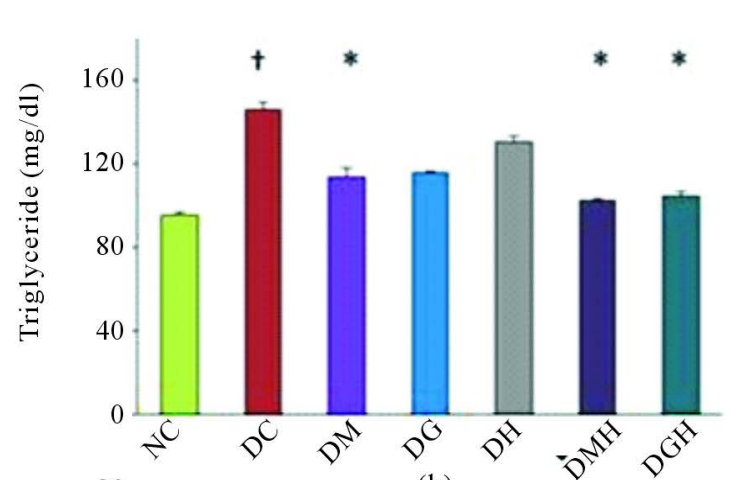

(b)

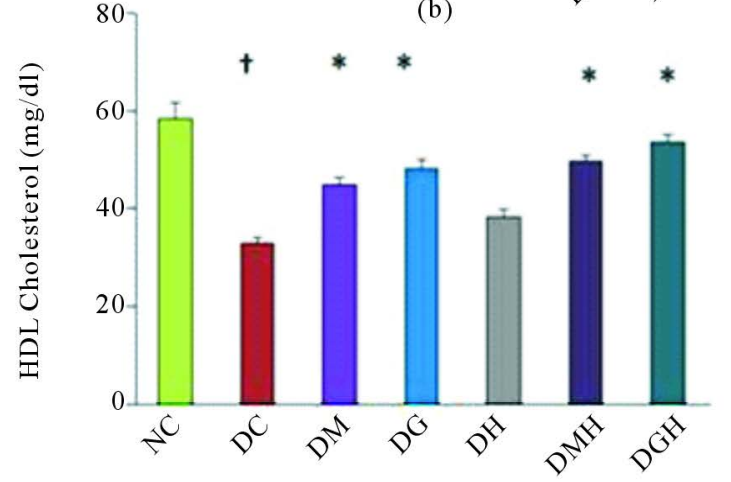

(d)

Figure 5. Effects of glibenclamide, metformin, HCQ and combination of drugs on lipid profile in alloxan induced diabetic rats after four weeks treatment. Changes in (a) TC level, (b) TG level, (c) LDL-C level and (d) HDL-C level. All values were presented as mean $\pm \mathrm{SEM} ; n=6$ in each group. ${ }^{\dagger} P<0.05$ compared with normal control group. $* P<0.05$ compared with diabetic control group. NC (Normal control), DC (Diabetic control), DG (Diabetic + Glibenclamide), DM (Diabetic + Metformin), DH (Diabetic + HCQ), DGH (Diabetic + Glibenclamide + HCQ), DMH (Diabetic + Metformin + HCQ).

of animals at the end of four weeks treatment period. Significant increase in TC level (40.24\%), TG level (52.89\%), LDL-Cholesterol level (48.09\%) was found and HDL-Cholesterol level (43.42\%) were reduced in alloxan induced diabetic rats in comparison with their respective normal rats after four weeks treatment as shown in Figures 5(a)-5(d).

Treatment with glibenclamide or metformin significantly $(P<0.05)$ decreased the TC level in diabetic rats. Combination of glibenclamide or metformin with HCQ further reduced the TC level in diabetic rats. No significant $(P<0.05)$ effect of HCQ alone in diabetic rats was observed.

Administration of metformin significantly $(P<0.05)$ decreased the levels of TG in diabetic rats compared to diabetic control rats. Besides, combination of metformin with HCQ further reduced the levels of TG in diabetic rats. On the other hand, glibenclamide and HCQ alone has no significant effect on TG but combination of glibenclamide with HCQ significantly $(P<0.05)$ decreased TG in diabetic rats.

Administration of glibenclamide or metformin significantly $(P<0.05)$ increased the levels of HDL-C but not HCQ alone in diabetic rats compared to diabetic control rats. Besides, combination of glibenclamide or metformin with HCQ further increasaed the levels of HDL cholesterol in diabetic rats.

Insignificantly decreased levels of LDL cholesterol were observed in diabetic control rats with the treatment of metformin, glibenclamide or HCQ alone in comparison with diabetic rats. But combination of HCQ with metformin or glibenclamide tended to bring LDL-C significantly $(P<0.05)$ towards normal values.

\subsection{Effects of Combination Therapy on the Level of Glycogen Content in Long-Term Alloxan Induced Diabetic Rats}

Figure 6 shows the results of liver glycogen level in different groups of animals at the end of four weeks treatment period. The liver glycogen level of the diabetic control rats were significantly $(P<0.05)$ lower $(45.39 \%)$ 


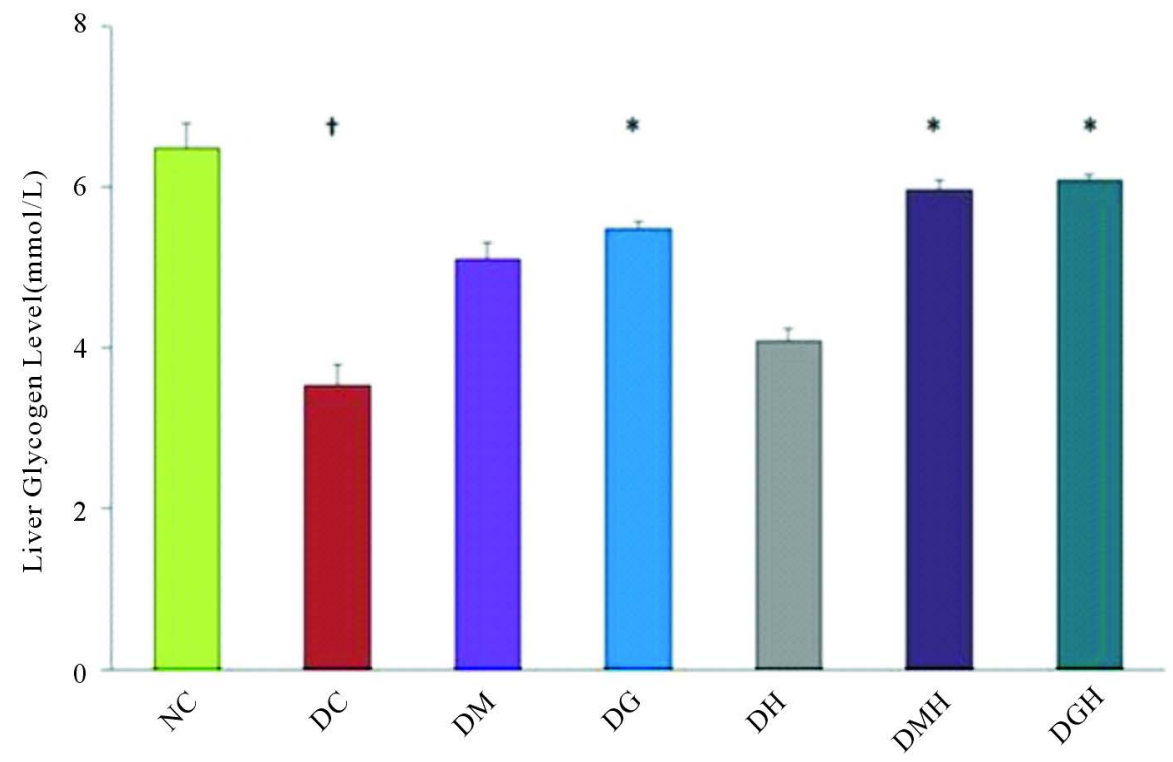

Figure 6. Effects of glibenclamide, metformin, HCQ and combination of drugs on liver glycogen level in alloxan induced diabetic rats after four weeks treatment. All values were presented as mean \pm SEM; $n=6$ in each group. ${ }^{\dagger} P<0.05$ compared with normal control group. $* P<0.05$ compared with diabetic control group. NC (Normal control), DC (Diabetic control), DG (Diabetic + Glibenclamide), DM (Diabetic + Metformin), DH (Diabetic + HCQ), DGH (Diabetic + Glibenclamide + HCQ), DMH (Diabetic + Metformin + HCQ).

than those of the normal control rats. Treatment with glibenclamide and metformin significantly $(P<0.05)$ increased the liver glycogen levels in alloxan induced diabetic rats (Figure 6). Combination of glibenclamide or metformin with HCQ further increased the liver glycogen level in diabetic rats. No significant effect of HCQ in diabetic rats was observed.

\section{Discussion}

Although the precise mechanism of alloxan induced diabetes remains unclear, there is increasing evidence that it involves the degeneration of islet cells by accumulation of cytotoxic free radicals [15]. Following its administration, alloxan is concentrated in the islets and in the liver, where it is reduced to dialuric acid. This acid is unstable in aqueous solutions and undergoes oxidation back to alloxan, accompanied by generation of $\mathrm{O}_{2}^{-}$, hydrogen peroxide and hydroxyl radicals by Fenton type reaction [15]. The liver contains high super oxide dismutase (SOD), catalase and glutathione peroxidase activities, which can scavenge these free radicals. On the contrary, the islet cells have low concentrations of these enzymes and are vulnerable to the cytotoxic effects of the free radicals. It is reported that increase in islet cell SOD activity can prevent or decrease alloxan toxicity [15].

Combination of HCQ with glibenclamide or metformin produced a significant decrease in the blood glucose level which is higher than that produced by either drug alone. Thus it seems likely that, apart from pancreatic action, HCQ may also possess extra-pancreatic action, which could have contributed to its hypoglycemic action. The mechanisms of action of glibenclamide and metformin are well documented [16] [17]. The mechanisms of hypoglycemia due to HCQ are inferred from studies of chloroquine (CQ), structurally similar anti-malarials. In-vitro evidence has shown that CQ reduces intracellular insulin degradation, increases intracellular insulin accumulation, slows receptor recycling and stimulates insulin-mediated glucose transport [18]. Chronic CQ treatment enhances insulin release in rats [19]. Animal data have shown that adding HCQ to alloxan induced diabetic rats led to a higher insulin level and lower glucose concentrations. HCQ may reduce cytosolic insulin metabolism [20] [21].

Combination of HCQ with metformin or glibenclamide significantly $(P<0.05)$ lowered the blood glucose level compared to that of metformin or glibenclamide alone. Moreover, the combination treatment caused significant improvement in the lipid profile as compared to that of either drug.

Diabetes is characterized by insulin deficiency which causes increased lipolysis in adipose tissues and in- 
creased entry of free fatty acids (FFA) to the liver [22]. Increased FFA and insulin independent glucose uptake in the liver is reported to stimulate triglyceride synthesis [23]. This increased synthesis of triglycerides tends to reduce protein content of lipoproteins, especially in very low-density lipoprotein (VLDL) and LDL while the triglyceride content increases [24]. This alteration in the protein and triglyceride compositions results in reduced uptake of these lipoproteins by their receptors [24]. As a result, dyslipidemia related to abnormalities in serum lipoproteins is an impending atherogenic risk factor in diabetes [25]. In this study, diabetic control rats showed significant changes in lipid abnormalities [22].

Metformin produces beneficial effects on the lipid profile mainly by correcting abnormal glucose metabolism [26]. It also produces moderate reduction in the triglyceride levels as a result of decreased hepatic synthesis of VLDL [27]. A similar observation has been reported in our study.

Glibenclamide improves lipid profile by reducing TC level and increasing HDL-C level. This could be explained on the basis that improvement in the lipid profile by glibenclamide in diabetic rats may be due to better glycemic control (i.e. secondary) [27]. Since glibenclamide acts by secondary mechanism the effect on the lipid profile produced by combination treatment is significantly more than that produced by glibenclamide alone.

Since the combination of HCQ with metformin and glibenclamide produced further improvement in the lipid profile than that produced by metformin or glibenclamide alone, it is suggested that HCQ may be acting by some different mechanism than that of metformin or glibenclamide on lipid metabolic pathways.

Induction of diabetes with alloxan was associated with decrease in hepatic glycogen, which could be attributed to the decrease in the availability of the active form of enzyme glycogen synthetase probably because of low levels of insulin. In the present study, HCQ with metformin or glibenclamide restored the depressed hepatic glycogen levels more effectively than the either drug alone possibly by increasing the level of insulin. Our results showed that supplementation of diabetic rats with combination therapy resulted in significant elevation in hepatic glycogen content. Decreased in the activities of the enzymes involved in glucose homeostasis in liver and kidney such as hexokinase has been reported in diabetic animals resulting in depletion of liver and muscle glycogen content [28]. Treatment with HCQ in combination with antidiabetic agents might increase the level of enzyme to the control level indicating an over-all increase in glucose influx. Our findings supported the findings of previously published reports [29] [30]. The exact mechanism of action needs further investigation.

HCQ, an antimalarial agent can offer a new and promising approach in the long-term management of maturity onset diabetes mellitus, because of its multifaceted action. Since it can produce a better glycemic control along with improvement in the lipid profile in animals, it is worthwhile to try HCQ in combination with other antidiabetic agents clinically.

\section{Conclusion}

Present study aimed to investigate the effect of HCQ in combination with glibenclamide or metformin on glycemic control in diabetic rats. Findings of this research indicate that the addition of HCQ to glibenclamide or metformin in alloxan induced diabetic rats help to reduce the serum glucose levels more significantly than that of glibenclamide or metformin alone. HCQ as an adjunct to glibenclamide or metformin leads to an improved glycemic control and some additional metabolic benefits not achieved with either glibenclamide or metformin alone. This study seems to suggest that the combination of HCQ with oral hypoglycemic agents may be a valuable adjuvant therapy to achieve and/or maintain glycemic control and possibly reduce or delay the onset of diabetic complications. A similar study in human subjects is desirable to determine if these results can be appropriately extrapolated to human diabetes. We need further study to determine the mechanism of action responsible for anti-diabetic activity.

\section{Acknowledgements}

The author(s) thank Square Pharmaceuticals Ltd, Bangladesh for its kind gift of glibenclamide and metformin. The author(s) also thank Dr. Abdul Alim Al Bari, Associate Professor, Department of Pharmacy, University of Rajshahi for his generous gift of hydroxychloroquine.

\section{References}

[1] The Diabetes Control and Complications Trial (DCCT) Research Group (1993) The Effect of Intensive Treatment of Diabetes on the Development and Progression of Long-Term Complications in the Diabetes Control in Insulin Depen- 
dent Diabetes Mellitus. The New England Journal of Medicine, 329, 977-986.

http://dx.doi.org/10.1056/NEJM199309303291401

[2] UK Prospective Diabetes Study (UKPDS) Group (1998) Intensive Blood-Glucose Control with Sulphonylureas or Insulin Compared with Conventional Treatment and Risk of Complications in Patients with Type 2 Diabetes. The Lancet, 352, 837-853. http://dx.doi.org/10.1016/S0140-6736(98)07019-6

[3] Turner, R.C., Cull, C.A., Frighi, V., et al. (1999) Glycemic Control with Diet, Sulfonylurea, Metformin, or Insulin in Patients with Type 2 Diabetes Mellitus: Progressive Requirement for Multiple Therapies. UK Prospective Diabetes Study (UKPDS) Group. The Journal of the American Medical Association, 281, 2005-2012. http://dx.doi.org/10.1001/jama.281.21.2005

[4] UK Prospective Diabetes Study Group (1995) UK Prospective Diabetes Study 16: Overview of 6 Years' Therapy of Type II Diabetes: A Progressive Disease. Diabetes, 44, 1249-1258. http://dx.doi.org/10.2337/diab.44.11.1249

[5] Yale, J.F., Valiquett, T.R., Ghazzi, M.N., et al. (2001) The Effect of a Thiazolidinedione Drug, Troglitazone, on Glycemia in Patients with Type 2 Diabetes Mellitus Poorly Controlled with Sulfonylurea and Metformin. A Multicenter, Randomized, Double-Blind, Placebo-Controlled Trial. Annals of Internal Medicine, 134, 737-745. http://dx.doi.org/10.7326/0003-4819-134-9_Part_1-200105010-00010

[6] Cook, M.N., Girman, C.J., Stein, P.P., et al. (2005) Glycemic Control Continues to Deteriorate after Sulfonylureas Are Added to Metformin among Patients with Type 2 Diabetes. Diabetes Care, 28, 995-1000. http://dx.doi.org/10.2337/diacare.28.5.995

[7] Robertson, R.P. (2004) Chronic Oxidative Stress as a Central Mechanism for Glucose Toxicity in Pancreatic Islet Beta Cells in Diabetes. The Journal of Biological Chemistry, 279, 42351-42354. http://dx.doi.org/10.1074/jbc.R400019200

[8] Robertson, R.P. and Harmon, J.S. (2006) Diabetes, Glucose Toxicity, and Oxidative Stress: A Case of Double Jeopardy for the Pancreatic Islet Beta Cell. Free Radical Biology and Medicine, 41, 177-184. http://dx.doi.org/10.1016/j.freeradbiomed.2005.04.030

[9] Grankvist, K., Marklund, S.L. and Taljedal, I.B. (1981) CuZn-Superoxide Dismutase, Mn-Superoxide Dismutase, Catalase and Glutathione Peroxidase in Pancreatic Islets and Other Tissues in the Mouse. Biochemical Journal, 199, 393-398.

[10] Kajimoto, Y. and Kaneto, H. (2004) Role of Oxidative Stress in Pancreatic Beta-Cell Dysfunction. Annals of the New York Academy of Sciences, 1011, 168-176. http://dx.doi.org/10.1196/annals.1293.017

[11] Emami, J., Pasutto, F.M., Mercer, J.R., et al. (1999) Inhibition of Insulin Metabolism by Hydroxychloroquine and Its Enantiomers in Cytosolic Fraction of Liver Homogenates from Healthy and Diabetic Rats. Life Sciences, 64, 325-335. http://dx.doi.org/10.1016/S0024-3205(98)00568-2

[12] Petri, M. (1996) Hydroxychloroquine Use in the Baltimore Lupus Cohort: Effects on Lipids, Glucose and Thrombosis. Lupus, 5, 16-22. http://dx.doi.org/10.1177/096120339600500105

[13] Wasko, M.C.M., Hubert, H.B. and Lingala, V.B. (2007) Hydroxychloroquine and Risk of Diabetes in Patients with Rheumatoid Arthritis. The Journal of the American Medical Association, 298, 187-193. http://dx.doi.org/10.1001/jama.298.2.187

[14] Iams, S.G. and Wexler, B.C. (1977) Alloxan Diabetes in Spontaneously Hypertensive Rats: Gravimetric, Metabolic and Histopathological Alterations. British Journal of Experimental Pathology, 58, 177-199.

[15] Halliwell, B. and Gutteridge, J.M.C. (1989) Free Radicals in Biology and Medicine. 2nd Edition, Clarendon Press, Oxford.

[16] Krentz, A.J. and Bailey, C.J. (2005) Oral Antidiabetic Agents: Current Role in Type 2 Diabetes Mellitus. Drugs, 65, 385-411. http://dx.doi.org/10.2165/00003495-200565030-00005

[17] Wiernsperger, N.F. and Bailey, C.J. (1999) The Antihyperglycaemic Effect of Metformin: Therapeutic and Cellular Mechanisms. Drugs, 58, 31-39. http://dx.doi.org/10.2165/00003495-199958001-00009

[18] Cynober, L., Aussel, C., Vaubourdolle, M., et al. (1987) Modulation of Insulin Action on 2-Deoxyglucose Uptake by Chloroquine in Chick Embryo Fibroblast. Diabetes, 36, 27-32. http://dx.doi.org/10.2337/diab.36.1.27

[19] Asamoah, K.A., Robb, D.A. and Furman, B.L. (1990) Chronic Chloroquine Treatment Enhances Insulin Release in Rats. Diabetes Research and Clinical Practice, 9, 273-278. http://dx.doi.org/10.1016/0168-8227(90)90056-Y

[20] Emami, J., Pasutto, F.M., Mercer, J.R., et al. (1998) Inhibition of Insulin Metabolism by Hydroxychloroquine and Its Enantiomers in Cytosolic Fraction of Liver Homogenates from Healthy and Diabetic Rats. Life Sciences, 64, 325-335. http://dx.doi.org/10.1016/S0024-3205(98)00568-2

[21] Emami, J., Gerstein, H.C., Pasutto, F.M. et al. (1999) Insulin Sparing Effect of Hydroxychloroquinein Diabetic Rats Is Concentration Dependent. Canadian Journal of Physiology and Pharmacology, 77, 118-123. 
http://dx.doi.org/10.1139/y98-146

[22] Ohno, T., Horio, F., Tanaka, S., et al. (2000) Fatty Liver and Hyperlipidemia in IDDM (Insulin-Dependent Diabetes Mellitus) of Streptozotocin-Treated Shrews. Life Sciences, 66, 125-131. http://dx.doi.org/10.1016/S0024-3205(99)00570-6

[23] Coppack, S.W., Jensen, M.D. and Miles, J.M. (1994) In Vivo Regulation of Lipolysis in Humans. Journal of Lipid Resesrch, 35, 177-193.

[24] Winocour, P.H., Durrington, P.N., Bhatnagar, D., et al. (1992) Abnormalities of VLDL, IDL, and LDL Characterize Insulin-Dependent Diabetes Mellitus. Arteriosclerosis, Thrombosis and Vascular Biology, 12, 920-928. http://dx.doi.org/10.1161/01.ATV.12.8.920

[25] Verges, B.L. (1999) Dyslipidemia in Diabetes Mellitus. Review of the Main Lipoprotein Abnormalities and Their Consequences on the Development of Atherogenesis. Diabetes and Metabolism, 25, 32-40.

[26] Defronzo, R.A. and Goodman, A.M. (1995) Efficacy of Metformin in Patient with Non-Insulin-Dependent Diabetes Mellitus. The New England Journal of Medicine, 333, 541-549. http://dx.doi.org/10.1056/NEJM199508313330902

[27] Chehade, J.M. and Mooradian, A.D. (2000) A Rational Approach to Drug Therapy of Type-2 Diabetes Mellitus. Drugs, 60, 95-113. http://dx.doi.org/10.2165/00003495-200060010-00006

[28] Grover, J.K., Vats, V. and Rathi, S.S. (2000) Antihyperglycemic Effect of Eugenia jambolana and Tinosporacardifolia in Experimental Diabetes and Their Effects on Key Metabolic Enzymes Involved in Carbohydrate Metabolism. Journal of Ethnopharmacology, 73, 461-470. http://dx.doi.org/10.1016/S0378-8741(00)00319-6

[29] Gold, A.H. (1970) The Effect of Diabetes and Insulin on Liver Glycogen Synthetase Activation. The Journal of Biological Chemistry, 245, 903-905.

[30] Goel, R.K., Mahajan, M.P. and Kulkarni, S.K. (2004) Evaluation of Antihyperglycemic Activity of Some Novel Monocyclic Beta Lactams. Journal of Pharmacy and Pharmaceutical Sciences, 7, 80-83. 
Scientific Research Publishing (SCIRP) is one of the largest Open Access journal publishers. It is currently publishing more than 200 open access, online, peer-reviewed journals covering a wide range of academic disciplines. SCIRP serves the worldwide academic communities and contributes to the progress and application of science with its publication.

Other selected journals from SCIRP are listed as below. Submit your manuscript to us via either submit@scirp.org or Online Submission Portal.
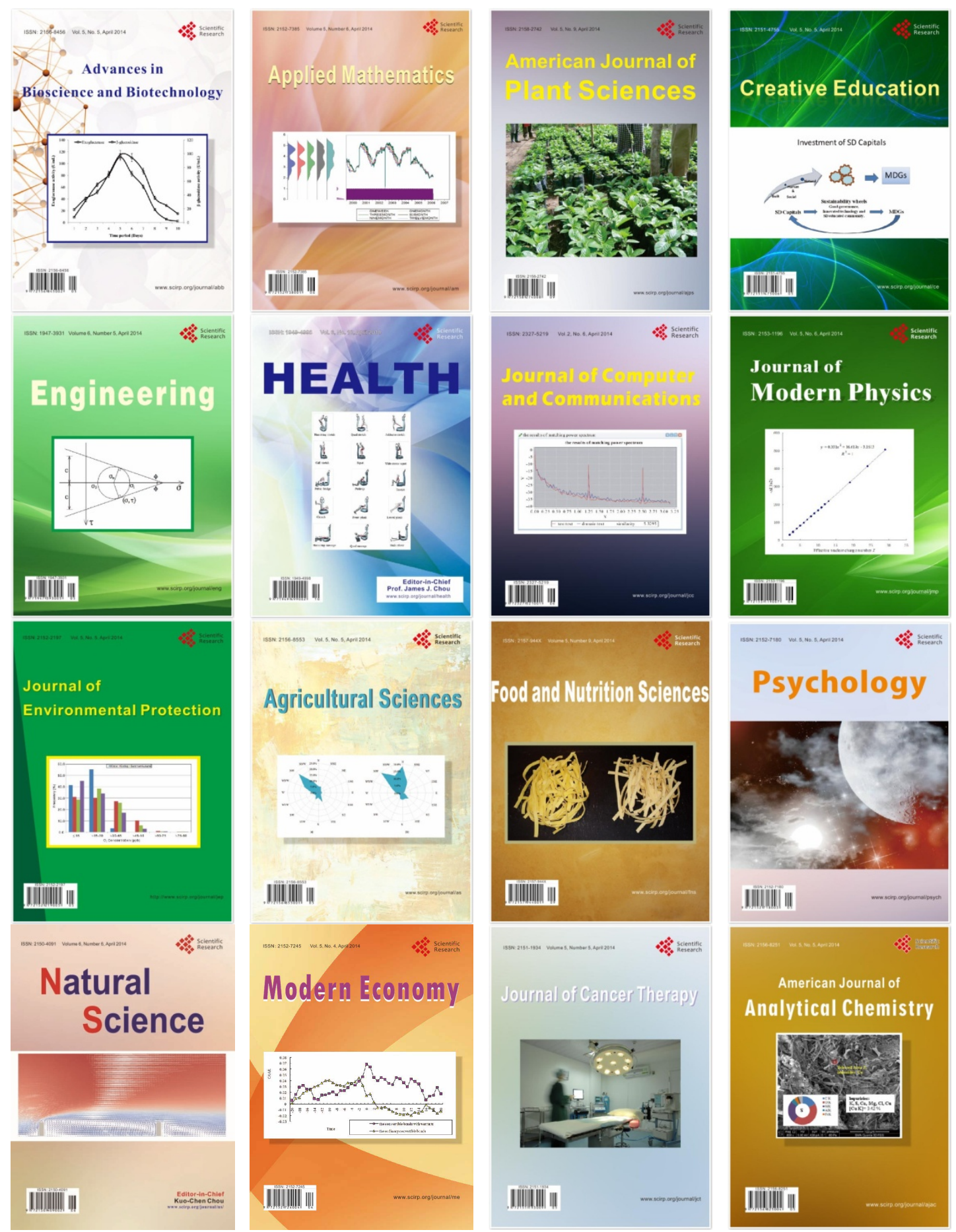